\title{
RESULTS ON THE NUCLEON SPIN STRUCTURE
}

\author{
Eva-Maria Kabuss * \\ Inst. für Kernphysik, Mainz University, D-55099 Mainz, Germany \\ FOR The Spin MuOn Collaboration
}

\begin{abstract}
SMC performed an investigation of the spin structure of the nucleon by measuring deep inelastic scattering of polarised muons off polarised protons and deuterons. Asymmetries and spin structure functions were obtained for $x>0.0008$ and $Q^{2}>0.2 \mathrm{GeV}^{2}$. Using a next-to-leading order QCD analysis of all experimental results polarised parton distributions and their first moments were determined. All data show a clear violation of the Ellis-Jaffe sum rule. The Bjorken sum rule is found to be valid and is tested to the $10 \%$ level.
\end{abstract}

\section{Introduction}

To investigate the spin structure of the nucleon SMC studies deep inelastic scattering of polarised muons off polarised protons and deuterons. From the measured longitudinal asymmetries the spin structure functions, $g_{1}$, can be extracted by

$$
A_{\|}=\frac{d \sigma^{\uparrow \downarrow}-d \sigma^{\uparrow \uparrow}}{d \sigma^{\uparrow \downarrow}+d \sigma^{\uparrow \uparrow}} \approx D \frac{g_{1}}{F_{1}} .
$$

The depolarisation factor $D$ gives the polarisation transfer from the lepton to the nucleon and increases with the energy transfer. $F_{1}$ is the unpolarised structure function.

In the quark parton modell (QPM) $g_{1}$ is related to the quark helicity distributions functions, $\Delta q=q^{\uparrow}-q^{\downarrow}$, by

$$
g_{1}\left(x, Q^{2}\right)=\frac{1}{2} \sum_{\mathrm{q}} e_{\mathrm{q}}^{2}\left(\Delta q\left(x, Q^{2}\right)+\Delta \bar{q}\left(x, Q^{2}\right)\right)
$$

where $Q^{2}$ is the negative square of the four momentum transfer, Bjorken $x$ the momentum fraction carried by the struck quark and $e_{\mathrm{q}}$ the quark charge. The notation $q^{\uparrow}\left(q^{\downarrow}\right)$ refers to parallel (antiparallel) orientation of the quark and the nucleon spins. The first moments $a_{\mathrm{q}}\left(Q^{2}\right)=\int_{0}^{1}\left(\Delta q\left(x, Q^{2}\right)+\Delta \bar{q}\left(x, Q^{2}\right)\right) \mathrm{d} x$ and $\Gamma_{1}\left(Q^{2}\right)=\int_{0}^{1} g_{1}\left(x, Q^{2}\right) \mathrm{d} x$ can be used to study the contribution of quark helicities to the nucleon spin and to test the Ellis-Jaffe [1] and Bjorken [2] sum rules.

\footnotetext{
*) supported by the BMBF

Czechoslovak Journal of Physics, Vol. O (2018), Suppl. S0
} 
The first measurement of the Ellis-Jaffe sum rule for the first moment of $g_{1}^{p}$ by the EMC [3] showed a strong violation of the sum rule. Interpreted in the QPM this pointed to a very small contribution of quark helicities to the proton spin and a negative polarisation of the strange sea quarks. This led to a series of new experiments at CERN, SLAC and also at DESY studying the spin structure of the proton and the neutron in more detail. These new data also allow to test the fundamental Bjorken sum rule $\Gamma_{1}^{\mathrm{p}}-\Gamma_{1}^{\mathrm{n}}=\left|\frac{g_{\mathrm{A}}}{g_{\mathrm{V}}}\right| / 6$. In addition, the structure function data are precise enough to perform next-to-leading order perturbative QCD analyses and determine the polarised parton distributions.

\section{The experiment}

The SMC experiment (NA37) was located at the M2 muon beam line of the CERN SPS and took data using polarised proton and deuteron targets from 1992 to 1996.

The 190 and $100 \mathrm{GeV}$ muon beam with about $4 \cdot 10^{7} \mu$ per $2.2 \mathrm{~s}$ spill was naturally polarised. The polarisation was determined by two independent measurements using a dedicated second spectrometer downstream of the main experiment. The first method measured the energy spectrum of the decay electrons from muon decay, the second one used the cross section asymmetry in polarised muon electron scattering 消. The results were $p_{\mu}=-0.795 \pm 0.019$ at $187.4 \mathrm{GeV}$ and $p_{\mu}=-0.81 \pm 0.03$ at $99.4 \mathrm{GeV}$.

The heart of the experiment was the polarised solid state target. It was polarised using dynamic nuclear polarisation in a $2.5 \mathrm{~T}$ longitudinal field of a superconducting magnet. The target temperature was maintained at $\approx 50-300 \mathrm{mK}$ during normal operation by a dilution refrigerator. From 1992 to 1995 SMC used butanol or deuterated butanol as target material whereas for the 1996 data taking ammonia was chosen improving the dilution factor $f$ by $30 \%$ from about 0.13 for butanol to about 0.17 for ammonia. The target was divided into two cells of $65 \mathrm{~cm}$ length polarised in opposite directions. To reduce systematic uncertainties due to changes in the detector response the target polarisation was reversed every 5 hours by rotating the magnetic field with the help of an additional $0.5 \mathrm{~T}$ dipole magnet. The average longitudinal polarisation was $p_{\mathrm{T}}= \pm 0.90$ for protons and $\pm 50 \%$ for deuterons. It was measured to an accuracy of 2-3\% using 9 NMR coils embedded in the target material. In addition to the continuous measurement of the proton polarisation the polarisation of ${ }^{14} \mathrm{~N}$ was determined in dedicated measurements [5] and found to depend on the proton polarisation as predicted by the equilibrium spin temperature relation. Typical nitrogen polarisations were \pm 0.14 for proton polarisations of \pm 0.89 .

The scattered muons and the produced forward going hadrons were detected in an open forward spectrometer using about 150 wire chamber planes placed upstream and downstream of a large gap dipol magnet. Due to the large number of wire planes the measured asymmetries were hardly sensitive to occasional problems 
with single planes. There was no particle identification for hadrons, but electron hadron separation was obtained with the help of a lead/iron scintillator calorimeter.

The measured counting rates for longitudinal beam and target polarisations were used to determine the raw asymmetry

$$
A_{\|}^{\mathrm{raw}}\left(x, Q^{2}\right)=\frac{N^{\uparrow \downarrow}-N^{\uparrow \uparrow}}{N^{\uparrow \downarrow}+N^{\uparrow \uparrow}},
$$

which is related to the lepton nucleon $\left(A_{\|}\right)$and photon nucleon asymmetries $\left(A_{1}\right.$, $A_{2}$ ) by

$$
A_{\|}^{\text {raw }}=p_{\mu} p_{\mathrm{T}} f A_{\|}=p_{\mu} p_{\mathrm{T}} f D\left(A_{1}+\eta A_{2}\right) .
$$

In a separate measurement using transversly polarised targets the transverse asymmetry

$$
A_{\perp}^{\text {raw }}=\frac{N^{\uparrow \rightarrow}-N^{\uparrow \leftarrow}}{N^{\uparrow \rightarrow}+N^{\uparrow \leftarrow}}=p_{\mu} p_{\mathrm{T}} f d\left(A_{2}+\eta^{\prime} A_{1}\right)
$$

was measured. $D, d, \eta$ and $\eta^{\prime}$ are known kinematic factors. $A_{2}$ was found to be small in the kinematic range of the SMC data [6,7] and thus the second term in eq. 4 can be neglected.

\section{Results for $A_{1}$ and $g_{1}$}

Inclusive asymmetries are determined using two methods. In the first one, called standard method, only scattered muons are used, in the second one, any hadron in addition to the scattered muon signals a true deep inelastic scattering and provides a discrimination between these events and background events like radiative events or elastic electron muon scattering [8]. Thus, the effective dilution factor $f^{\prime}=f \cdot \sigma^{1 \gamma} / \sigma^{\text {tot }}$ is much larger for the hadron method than for the standard one (see fig. 17a).

In the SMC spectrometer mainly forward produced charged hadrons with momenta $p_{\mathrm{h}}>5 \mathrm{GeV}$ were detected. Events with a high mass $W$ of the final state contain in general several hadrons with high momenta. High $W$ events are typically found at small $x$, so that a good acceptance for deep inelastic events is obtained for $x<0.1$, whereas in the high $x$ region many hadrons are not detected due to the low momenta at low $W$. In addition cuts are applied to reject electrons from conversion of Bremstrahlung photons. Monte Carlo studies show that more than $80 \%$ of the deep inelastic events are found by requiring at least one additional hadron to the scattered muon. The results for $A_{1}^{\mathrm{p}}$ from both methods are compared in fig. 1 $1 \mathrm{~b}$ for $x>0.0008$ and $Q^{2}>0.2 \mathrm{GeV}^{2}$. Good agreement is obtained between the two results with the statistical erros bars smaller for $x<0.1$ for the hadron method. The optimal SMC data set for $A_{1}^{\mathrm{p}}$ and $A_{1}^{\mathrm{d}}$ is shown in fig. 2 2 in comparison to measurements from the EMC [3] at a similar $Q^{2}$ and the lower $Q^{2}$ data from E143 9]. Systematic uncertainties are shown as bands at the bottom. At low $x$, the main contributions are due to the uncertainty of radiative corrections, the neglect of $A_{2}$

Czech. J. Phys. 0/S0 (2018) 

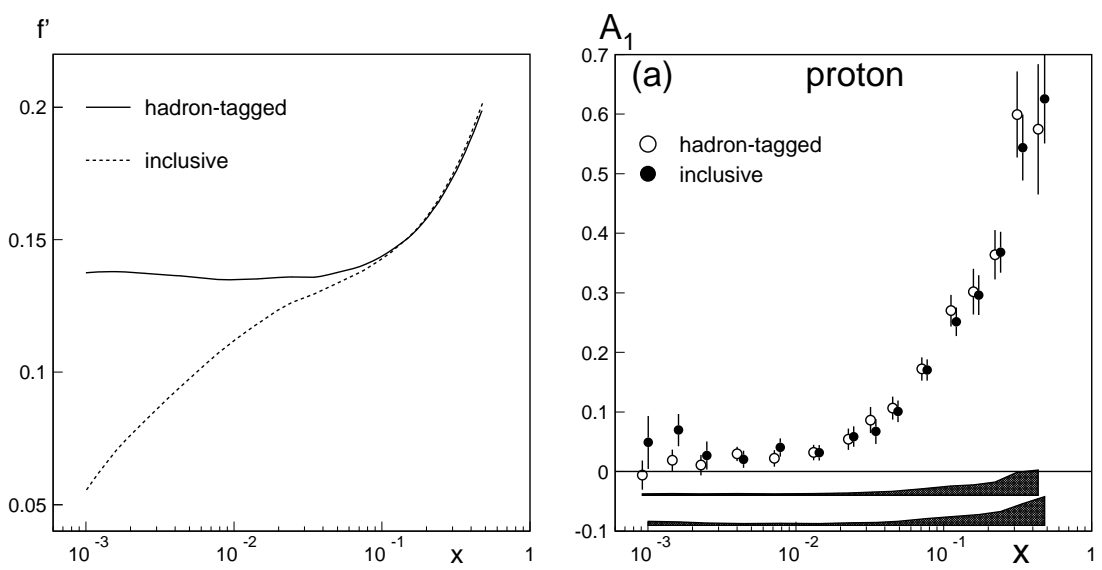

Fig. 1. Comparison a) of the effective dilution factor $f^{\prime}, \mathrm{b}$ ) of the proton asymmetries $A_{1}^{\mathrm{p}}$ from the standard analysis method and the hadron method.

and acceptance variations. At high $x$ they stem from uncertainties of $R$ and the beam and target polarisation.
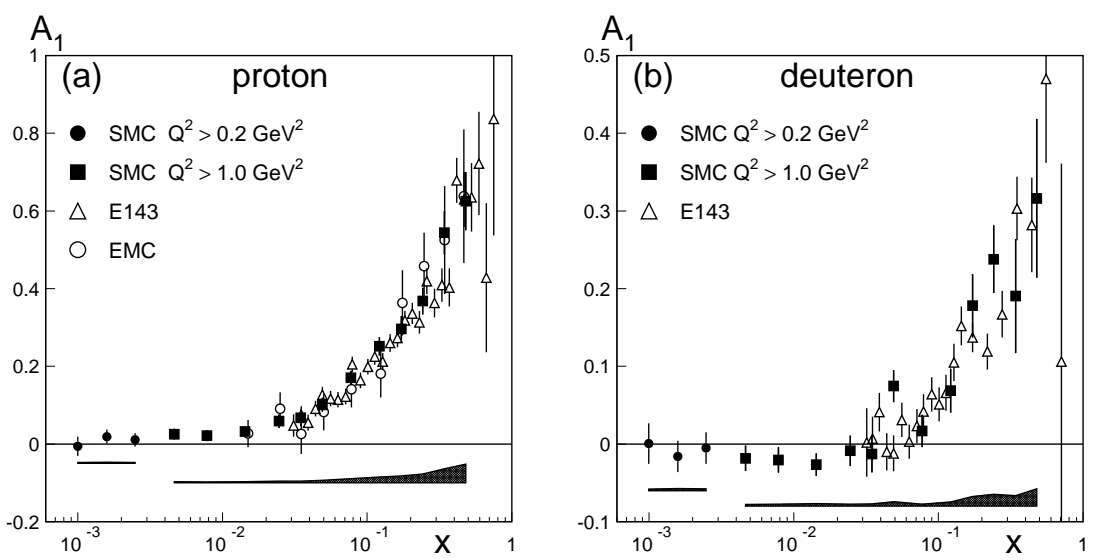

Fig. 2. The optimal data set of SMC for $A_{1}$ together with results from other experiments. Statistical errors are shown as error bars, while the shaded bands below indicate the systematic uncertainty for the SMC measurement.

In addition to the $x$ dependence also the $Q^{2}$ dependence of $A_{1}$ was investigated. No $Q^{2}$ dependence is observed in the kinematic range of the SMC data.

An extension of the measured asymmetries down to $x=6 \cdot 10^{-5}$ with $Q^{2}>$ $0.01 \mathrm{GeV}^{2}$ is under study. In this kinematic region the inclusive events are domi- 
nated by muon scattering off atomic electrons. In 1995/6 data were collected using a dedicated "low $x$ " trigger in which both a minimal energy deposit in the hadronic part of the calorimeter and the detection of a muon was demanded [10]. Fig. 3a shows the kinematic acceptance of this trigger compared to other experiments, while the preliminary results for $A_{1}^{\mathrm{p}}$ are shown in fig. $3 \mathrm{p}$. The two SMC data sets are compatible in the overlap region. At low $x$ no significant spin effects are visible.
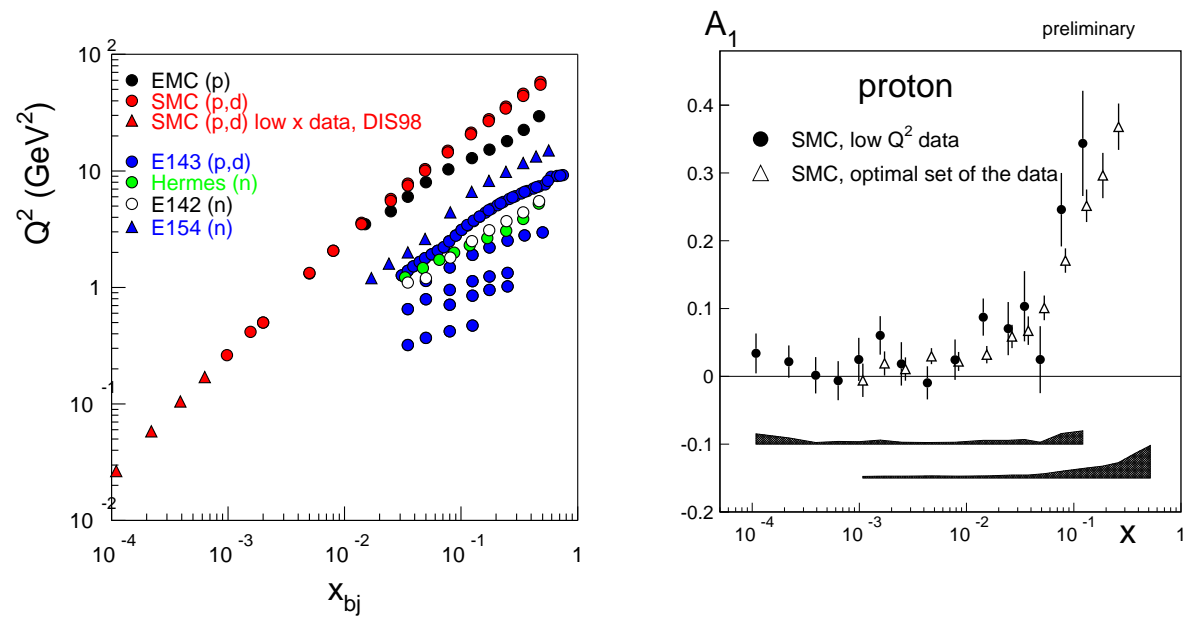

Fig. 3. a) Kinematic acceptance of the "low $x$ "trigger compared to other experiments, b) preliminary results on $A_{1}^{\mathrm{p}}$ compared to the SMC optimal data set. The error bars are statistical errors, the bands indicate the systematic uncertainties.

For $Q>0.2 \mathrm{GeV}^{2}$ the spin structure functions, $g_{1}$, are calculated from the measured asymmetries with eq. 11 using $F_{1}=F_{2} / 2 x(1+R)$, where $F_{2}\left(x, Q^{2}\right)$ and

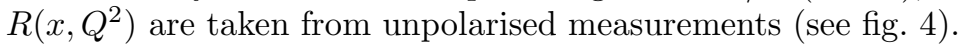

\section{QCD analysis}

To determine the first moment, $\Gamma_{1}\left(Q_{0}^{2}\right)$, the results for $g_{1}\left(x, Q^{2}\right)$ at a fixed $Q^{2}=Q_{0}^{2}$ are needed. Though the precision of the available data and the $Q^{2}$ range do not allow a direct measurement of a possible $Q^{2}$ dependence of $A_{1}=g_{1} / F_{1}$ different $Q^{2}$ behaviours of $g_{1}$ and $F_{1}$ are expected from perturbative QCD. Therefore, the $Q^{2}$ dependence of $g_{1}$ is estimated from a next-to-leading-order (NLO) perturbative QCD analysis 11] of all available proton, neutron and deuteron data with $Q^{2}>$ $1 \mathrm{GeV}^{2}$. In this analysis $g_{1}$ is decomposed into polarised quark singlet $\Delta \Sigma$, gluon $\Delta g$ and nonsinglet distributions $\Delta q^{\mathrm{NS}}$ for the proton and the neutron. The NLO analysis is done in the Adler-Bardeen and the $\overline{\mathrm{MS}}$ scheme [12]. In the fit some parameters like the normalisation of the nonsinglet distributions were constrained by using the neutron and hyperon $\beta$ decay constants assuming SU(3) flavour. The

Czech. J. Phys. 0/S0 (2018) 

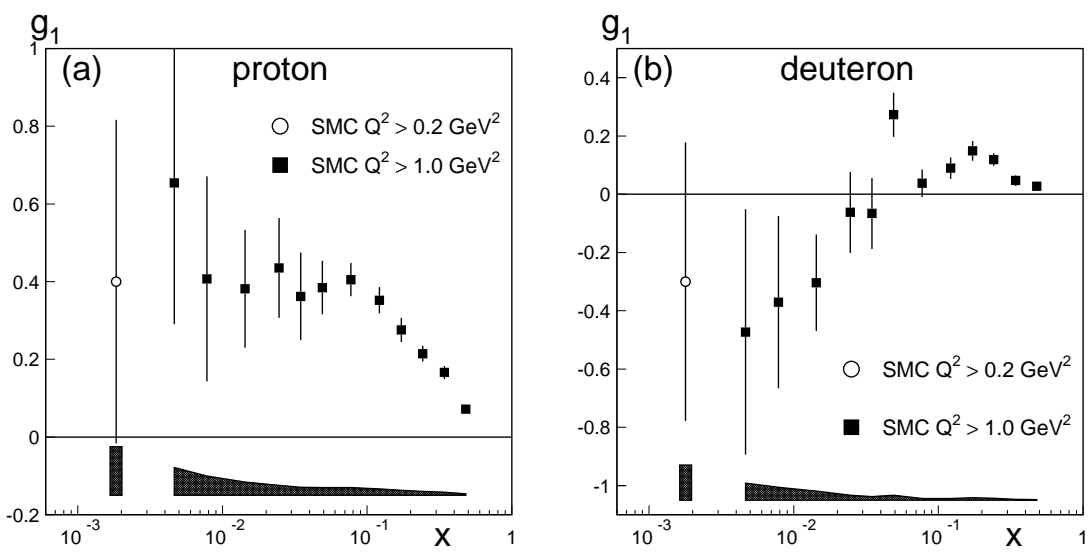

Fig. 4. a) $g_{1}^{\mathrm{p}}$ and b) $g_{1}^{\mathrm{d}}$ vs. $x$ at the measured $Q^{2}$ for the optimal data set. The statistical uncertainties are shown as error bars, and the bands indicate the size of the systematic uncertainties.

results for the polarised parton distributions $x \Delta \Sigma, x \Delta g$ and $x \Delta q^{\mathrm{NS}}$ are shown in fig. 司.

In the QPM the quark contribution to the nucleon spin is given by the singlet axial charge $a_{0}=a_{\mathrm{u}}+a_{\mathrm{d}}+a_{\mathrm{s}}$ whereas it is ambiguous in NLO pQCD due to the axial anomaly. Thus, the first moment of $\Delta \Sigma, a_{\Sigma}$ is equal to $a_{0}$ in the $\overline{\mathrm{MS}}$ scheme, while in the $\mathrm{AB}$ scheme the gluon contribution must be subtracted. The results $a_{0}^{\mathrm{AB}}=0.23 \pm 0.07$ (sta) \pm 0.19 (sys\&th) and $a_{0}^{\overline{\mathrm{MS}}}=0.19 \pm 0.05($ sta $) \pm 0.04$ (sys\&th) are similar in both schemes and correspond to about $1 / 3$ of the naive Ellis-Jaffe expectation of 0.58 . The result for the gluon contribution $a_{\mathrm{g}}\left(Q^{2}\right)=\int_{0}^{1} \Delta g\left(x, Q^{2}\right) \mathrm{d} x$

$$
a_{\mathrm{g}}^{\mathrm{AB}}=0.99{ }_{-0.31}^{+1.17}(\mathrm{sta}){ }_{-0.22}^{+0.42}(\mathrm{sys}){ }_{-0.45}^{+1.43}(\mathrm{th})
$$

shows that very little can be said about this quantity on the basis of the present data.

\section{Sum rules}

Using the results of the QCD analysis $g_{1}\left(x, Q_{0}^{2}=5 \mathrm{GeV}^{2}\right)$ is obtained from $g_{1}\left(x, Q_{0}^{2}\right)=g_{1}\left(x, Q^{2}\right)+\left[g_{1}^{\mathrm{fit}}\left(x, Q_{0}^{2}\right)-g_{1}^{\mathrm{fit}}\left(x, Q^{2}\right)\right]$. The extrapolation of $g_{1}$ to $x=0$ and $x=1$ is done by integrating the fit results for $x<0.003$ and $x>0.8$ (see fig. $6 \mathrm{a}$ ). The results at $Q_{0}^{2}=5 \mathrm{GeV}^{2}$

$$
\begin{aligned}
& \Gamma_{1}^{\mathrm{p}}=0.121 \pm 0.003(\mathrm{sta}) \pm 0.005(\mathrm{sys}) \pm 0.017(\mathrm{th}) \\
& \Gamma_{1}^{\mathrm{d}}=0.021 \pm 0.004(\mathrm{sta}) \pm 0.003(\mathrm{sys}) \pm 0.016(\mathrm{th}) \\
& \Gamma_{1}^{\mathrm{n}}=-0.075 \pm 0.007(\mathrm{sta}) \pm 0.005(\mathrm{sys}) \pm 0.019(\mathrm{th})
\end{aligned}
$$



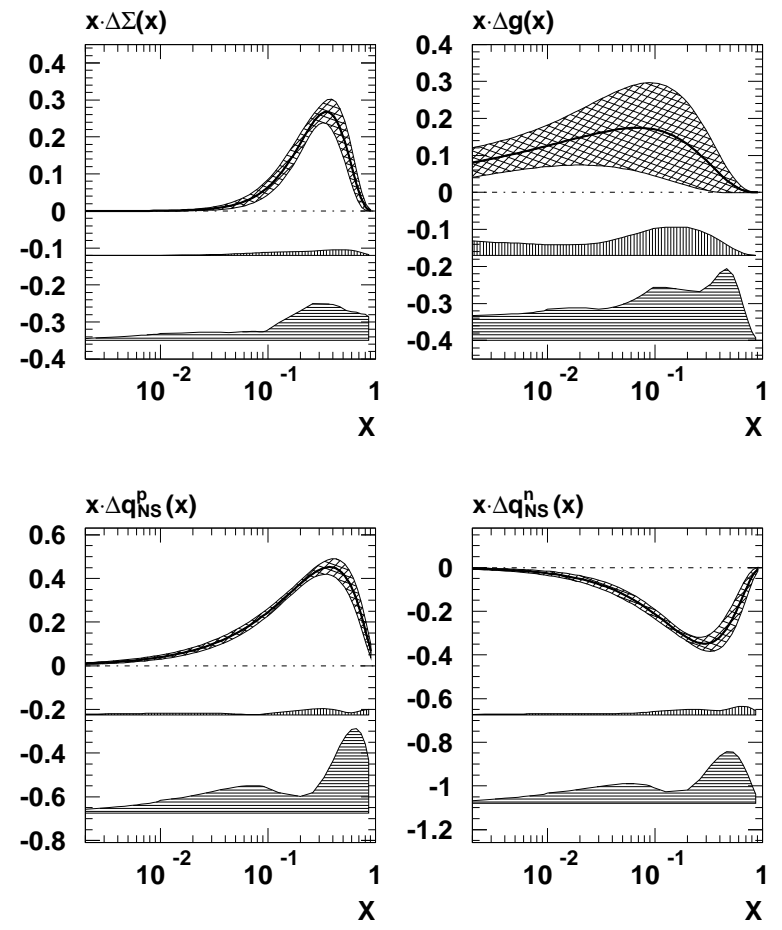

Fig. 5. Polarised parton distributions determined at $Q^{2}=1 \mathrm{GeV}^{2}$. The uncertainties are statistical (cross hatched band), experimental systematic (vertically hatched band), and theoretical (horizontally hatched band).

show a violation of the Elle-Jaffe sum rules of about $3 \sigma$.

To test the Bjorken sum rule the fit has to be repeated releasing the constraints for the nonsinglet moments using $g_{\mathrm{A}} / g_{\mathrm{V}}$ as a free parameter. A consistent value of $g_{\mathrm{A}} / g_{\mathrm{V}}$ with the nominal value used above is obtained. The resulting value for the Bjorken sum rule at $Q^{2}=5 \mathrm{GeV}^{2}$

$$
\Gamma_{1}^{\mathrm{p}}-\Gamma_{1}^{\mathrm{n}}=0.174 \stackrel{+0.024}{+0.012}
$$

is in excellent agreement with the theoretical value of $\Gamma_{1}^{\mathrm{p}}-\Gamma_{1}^{\mathrm{n}}=0.181 \pm 0.003$. An alternative way to determine $\Gamma_{1}^{\mathrm{p}}-\Gamma_{1}^{\mathrm{n}}$ restricts the QCD analysis to the nonsinglet distribution which is decoupled from the evolution of $\Delta \Sigma$ and $\Delta g$. Here, data points for $g_{1}^{\mathrm{p}}$ and $g_{1}^{\mathrm{n}}$ at the same kinematics are needed so that only the SLAC E143 and SMC can be used presently (see fig. 6b)). The result

$$
\Gamma_{1}^{\mathrm{p}}-\Gamma_{1}^{\mathrm{n}}=0.181 \stackrel{+0.026}{+0.021}
$$

Czech. J. Phys. 0/S0 (2018) 

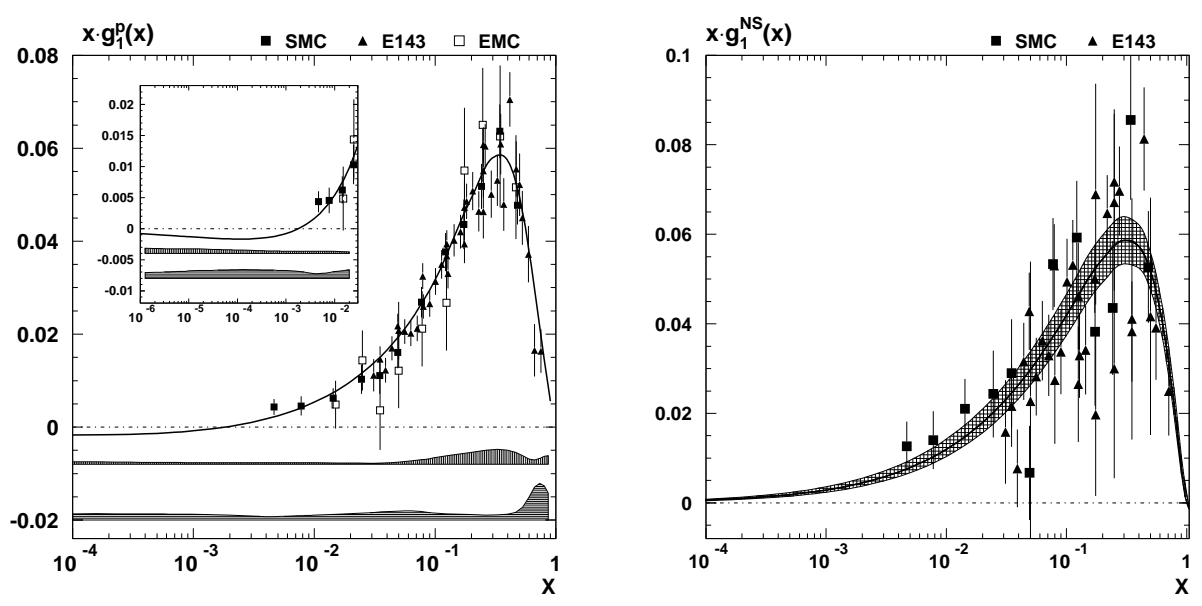

Fig. $6 . \quad$ a) $x g_{1}^{\mathrm{p}}$ vs. $x$ for the world data with the uncertainties of the fit due to experimental systematics and theoretical sources shown by the bands, b) $x g_{1}^{\text {NS }}$ vs. $x$ from E143 and SMC with the QCD fit at $Q^{2}=5 \mathrm{GeV}^{2}$ with the error band representing the systematic and theoretical uncertainty of the fit. All points are shown with their statistical errors.

is constistent with the above result, but due to the smaller data set the experimental errors are larger. The theoretical error, however, is smaller than for the first method, so that with the forthcoming data from E155 and HERMES a significant improvement is expected.

\section{References}

[1] J. Ellis and R. L. Jaffe, Phys. Rev. D9 (1974) 1444; Phys. Rev. D10 (1974) 1669.

[2] J. D. Bjorken, Phys. Rev. 148 (1966) 1467; Phys. Rev. D1 (1970) 1376.

[3] EMC, J. Ashman et al., Nucl. Phys. B328 (1989) 1.

[4] SMC, B. Adeva et al., Nucl. Instr. Meth. A343 (1994) 363, B. Adeva et al., in preparation.

[5] SMC, B. Adeva et al., Nucl. Instr. Meth. A419 (1998) 60.

[6] SMC, D. Adams et al., Phys. Lett. B296 (1997) 338.

[7] SMC, D. Adams et al., Phys. Rev. D56 (1997) 5330.

[8] SMC, B. Adeva et al., CERN-EP/98-85, submitted to Phys. Rev. D.

[9] E143, K. Abe et al., Phys. Rev. Lett. 74 (1995) 346.

[10] J. Kiryluk, Proc. of DIS98 (Brussels 1998), to be published.

[11] SMC, B. Adeva et al., CERN-EP/98-86, submitted to Phys. Rev. D.

[12] R. Ball, S. Forte and G. Ridolfi, Phys. Lett. B378 (1996) 255. 